九州大学学術情報リポジトリ

Kyushu University Institutional Repository

内因性トリプトファン光産物FICZはTGF- $\beta$ シグナル

によるコラーゲン合成を阻害することで光老化に関

与する

村井，美華

http://hdl. hand le. net/2324/2236066

出版情報：Kyushu University，2018，博士（医学），課程博士 バージョン：

権利関係 : 


\title{
An endogenous tryptophan photo-product, FICZ, is potentially involved in photo-aging by reducing TGF- $\beta$-regulated collagen homeostasis
}

\author{
Mika Murai $^{\mathrm{a}}$, Gaku Tsuji ${ }^{\mathrm{a}}$, Akiko Hashimoto-Hachiya ${ }^{\mathrm{a}}$, Yoshihito Kawakami ${ }^{\mathrm{b}}$, \\ Masutaka Furue $^{\mathrm{a}, \mathrm{b}, \mathrm{c}}$, Chikage Mitoma ${ }^{\mathrm{a}, \mathrm{b}, *}$ \\ a Department of Dermatology, Graduate School of Medical Sciences, Kyushu University, Fukuoka 812-8582, Japan \\ ${ }^{\mathrm{b}}$ Research and Clinical Center for Yusho and Dioxin, Kyushu University Hospital, Fukuoka 812-8582, Japan \\ ${ }^{\mathrm{c}}$ Division of Skin Surface Sensing, Department of Dermatology, Graduate School of Medical Sciences, Kyushu University, Fukuoka 812-8582, Japan
}

\section{A R T I C L E I N F O}

\section{Article history:}

Received 14 June 2017

Received in revised form 23 September 2017 Accepted 6 October 2017

\section{Keywords:}

6-Formylindolo[3,2-b]carbazole

FICZ

Photo-aging

Ultraviolet

Dermal fibroblast

Aryl hydrocarbon receptor

\begin{abstract}
A B S T R A C T
Background: Persistent ultraviolet (UV) radiation in the form of sunlight causes photo-aging of the skin by reducing the production of type I collagen, the major constituent of the extracellular matrix of the dermis. Transforming growth factor (TGF)- $\beta$ transforms dermal fibroblasts into $\alpha 2$-smooth muscle actin (ACTA2)-expressing myofibroblasts. Myofibroblasts produce a precursor form of type I collagen, type I procollagen (collagen I), consisting of pro-alpha1 (produced by the COL1A1 gene) and pro-alpha2 chains (produced by the COL1A2 gene). Smad2/3 is a key downstream molecule of TGF- $\beta$ signaling. The mechanisms through which UV inhibits collagen I synthesis are not fully understood. 6-Formylindolo $[3,2-b]$ carbazole (FICZ) is an endogenous tryptophan photo-metabolite generated by UV irradiation. FICZ is well known as a high-affinity ligand for aryl hydrocarbon receptor (AHR). However, the physiological roles of FICZ in photo-aging have yet to be addressed.

Objective: To evaluate the effects of FICZ on the TGF- $\beta$-mediated ACTA2 and collagen I expression in normal human dermal fibroblasts (NHDFs).

Methods: Quantitative real-time polymerase chain reaction and western blot analysis were performed to determine the expression of ACTA2, COL1A1, and COL1A2 in NHDFs with or without FICZ and TGF- $\beta$. The phosphorylated Smad2/3 (pSmad2/3) protein levels in cytoplasmic or nuclear portions were investigated by western blot analysis. Immunofluorescence staining was conducted to evaluate pSmad2/3 localization, and F-actin staining with phalloidin was performed to visualize actin polymerization in myofibroblasts. The actions of FICZ on the TGF- $\beta$-mediated collagen I expression and nuclear translocation of $\mathrm{pSmad} 2 / 3$ were analyzed in the presence of selective AHR antagonists or in AHR-knockdown NHDFs.

Results: We found that FICZ significantly inhibited the TGF- $\beta$-induced upregulation of mRNA and protein levels of ACTA2 and collagen I and actin polymerization in myofibroblasts. FICZ did not disturb the phosphorylation of Smad2/3. Notably, FICZ reduced the expression of pSmad2/3 in the nucleus, while it increased that in the cytoplasm, suggesting that it inhibits the nuclear translocation of $\mathrm{pSmad} 2 / 3$ induced by TGF- $\beta$. The inhibitory actions of FICZ on the TGF- $\beta$-mediated collagen I expression and nuclear translocation of $\mathrm{pSmad} 2 / 3$ were independent of AHR signaling. Another endogenous AHR agonist, kynurenine, also inhibited the TGF- $\beta$-mediated ACTA2 and collagen I upregulation in NHDFs in an AHRindependent manner; however, its effects were insignificant in comparison with those of FICZ. Conclusions: These findings suggest that the endogenous photo-product FICZ may be a key chromophore that involves in photo-aging. Downregulation of FICZ signaling is thus a potential strategy to protect against photo-aging.
\end{abstract}

(c) 2017 Japanese Society for Investigative Dermatology. Published by Elsevier Ireland Ltd. All rights reserved.

\footnotetext{
Abbreviations: FICZ, formylindolo[3,2- $b$ ]carbazole; AHR, aryl hydrocarbon receptor; NHDF, normal human dermal fibroblast; ROS, reactive oxygen species; ACTA2, $\alpha 2-$ smooth muscle actin; ITE, 2-(1'H-indole-3'-carbonyl)-thiazole-4-carboxylic acid methyl ester; pSmad2/3, phosphorylated Smad2/3.

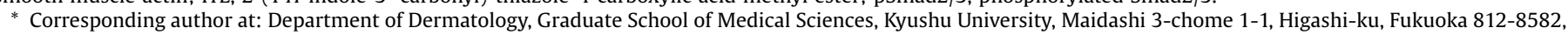
Japan.

E-mail addresses: mika-m@dermatol.med.kyushu-u.ac.jp (M. Murai), gakku@dermatol.med.kyushu-u.ac.jp (G. Tsuji), ahachi@dermatol.med.kyushu-u.ac.jp

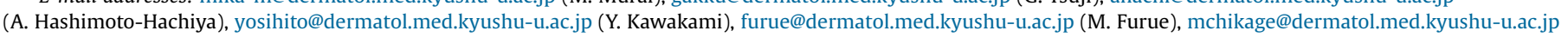
(C. Mitoma).
} 


\section{Introduction}

Ultraviolet (UV) radiation has major adverse effects on the outermost layer of the body, namely, the skin. The mechanisms by which UV causes photocarcinogenesis and photo-aging are well established. UV-mediated responses directly induce deleterious cell damage by generating DNA photo-products in the nucleus, namely, reactive oxygen species (ROS) and organic free radicals [1-4]. A variety of chromophores and photosensitizers have been reported to be produced in the skin in response to UV exposure $[5,6]$. However, the responsible chromophores that contribute to cutaneous photo-aging have not been fully identified. The L-tryptophan metabolite 6-formylindolo[3,2-b] carbazole (FICZ) was identified as a UVB-driven photo-product in human HaCaT keratinocytes [7]. FICZ also acts as a strong photosensitizer that enhances UVA-induced oxidative stress in a coordinated manner [8]. Notably, FICZ is a high-affinity ligand for the aryl hydrocarbon receptor (AHR) that is a constitutively expressed chemosensor for halogenated polycyclic aromatic hydrocarbons and their related xenobiotics [7,9-11]. Other endogenous AHR ligands have also been identified, including kynurenine and 2-( $1^{\prime} H$-indole-3'-carbonyl)-thiazole-4-carboxylic acid methyl ester (ITE) [12,13]. Upon ligand binding, the cytoplasmic AHR translocates into the nucleus and induces the transcription of xenobiotic-metabolizing enzymes such as cytochrome P450 (CYP)1A1 and CYP1B1 [10,14-18]. AHR signaling is an integral part of the UV responses because UV exposure and FICZ upregulate the CYP1A1 and CYP1B1 expression in human keratinocytes, and their upregulation is canceled by AHR deficiency or a selective AHR antagonist [19-21]. Moreover, UV-induced ROS production was shown to be downregulated in AHR-knockdown keratinocytes [22].

Type I collagen is the major constituent of the extracellular matrix of the dermis and is very important for the maintenance of skin integrity [23]. A precursor form of it, type I procollagen, consists of two pro-alpha 1 chains encoded by the COL1A1 gene and one pro-alpha2 chain encoded by the COL1A2 gene [24]. After type I procollagen has been processed by enzymes, mature type I collagen fibers are formed. Upon cutaneous injury, dermal fibroblasts migrate into the damaged area and differentiate into myofibroblasts via the effects of various growth factors and cytokines that are released from surrounding tissue and immune cells that have migrated there [25-27]. Transforming growth factor (TGF)- $\beta$, one of the most potent profibrotic cytokines, actively induces the expression of $\alpha 2$-smooth muscle actin (ACTA2) with the assembly of actin stress fibers, promoting the fibroblast-tomyofibroblast transition $[25,28]$. The myofibroblasts produce abundant type I procollagen $[25,26]$. In photo-damaged skin, the amounts of collagen are significantly decreased [29]. UVB irradiation impairs the TGF- $\beta$-mediated production of type I procollagen from normal human dermal fibroblasts (NHDFs) $[30,31]$. However, the effects of the photo-product FICZ on NHDFs are not fully understood.

In this study, we found that FICZ inhibits the TGF- $\beta$-induced ACTA2 and collagen I expression as well as the TGF- $\beta$-induced transformation of fibroblasts into myofibroblasts. The present results highlight the potential involvement of FICZ as an endogenous target molecule for preventing photo-aging.

\section{Materials and methods}

\subsection{Reagents and antibodies}

TGF- $\beta$ (R\&D Systems, Minneapolis, MN), FICZ (Enzo Life Sciences, Exeter, UK), and L-kynurenine (Sigma-Aldrich, St. Louis, MO) were used in this study. The selective AHR antagonists $\mathrm{CH}-$
223191 and GNF351 were obtained from Sigma-Aldrich and Merck Millipore (Billerica, MA) respectively. For western blot analysis, an anti-human ACTA2 mouse monoclonal antibody (A2547; SigmaAldrich), anti-collagen I rabbit monoclonal antibody (ab138492; Abcam, Cambridge, MA), anti-Smad2/3 rabbit polyclonal antibody (\#5678; Cell Signaling Technology, Danvers, MA), anti-phosphorylated Smad2 (Ser465/Ser467)/Smad3 (Ser423/Ser425) (pSmad2/ 3) rabbit monoclonal antibody (\#8828; Cell Signaling Technology), anti-histone deacetylase 1 (HDCA1) rabbit polyclonal antibody (ab7028; Abcam), and anti- $\alpha$-tubulin mouse monoclonal antibody (sc5286; Santa Cruz Biotechnology, Dallas, TX) were used. An antiAHR rabbit polyclonal antibody (sc-5579; Santa Cruz Biotechnology) was used for both western blotting and immunofluorescent staining. An anti-pSmad2/3 goat polyclonal antibody (sc11769; Santa Cruz Biotechnology) was used for immunofluorescent staining. Normal rabbit and goat IgGs were purchased from Santa Cruz Biotechnology. Anti- $\beta$-actin mouse monoclonal antibody (\#3700) and horseradish peroxidase (HRP)-linked anti-mouse IgG (\#7076S) and anti-rabbit IgG (\#7074S) were purchased from Cell Signaling Technology.

\subsection{Cell culture}

NHDFs obtained from Lonza (Walkersville, MD) and the human keratinocyte cell line $\mathrm{HaCaT}$ were grown in a culture flask at $37^{\circ} \mathrm{C}$ in $5 \% \mathrm{CO}_{2}$ using Dulbecco's Modified Eagle's Medium (DMEM; Sigma-Aldrich) supplemented with inactivated $5 \%$ fetal bovine serum, Modified Eagle's Medium Non-Essential Amino Acids (MEM NEAA), $10 \mathrm{mM}$ HEPES, and $1 \mathrm{mM}$ sodium pyruvate (Thermo Fisher Scientific, Waltham, MA). The culture medium was replaced every 2 days. Near-confluent (70\%-90\%) cells were disaggregated with $0.05 \%$ trypsin $/ 0.01 \%$ ethylenediamine tetraacetic acid and subcultured. NHDFs that had undergone fewer than six passages were used in all of the experiments.

NHDFs $\left(2.5 \times 10^{5}\right)$ or HaCaT cells were seeded in six-well culture plates, allowed to attach for $48 \mathrm{~h}$, and subsequently treated with FICZ, TGF- $\beta$, or L-kynurenine. FICZ was dissolved in dimethyl sulfoxide (DMSO) at a concentration of 0.5 or $5 \mathrm{mM}$ and was further diluted in the culture medium. Various concentrations of FICZ (0.01-400 nM), TGF- $\beta$ ( $0.1-5 \mathrm{ng} / \mathrm{ml})$, and L-kynurenine were prepared in the culture medium. Control culture medium contained comparable concentrations of DMSO (up to $0.02 \%$ ).

\subsection{Transfection of AHR SiRNA}

AHR siRNA (s1200) and siRNA consisting of a scrambled sequence that would not lead to specific degradation of any cellular mRNA (control siRNA) were purchased from Ambion (Austin, TX). NHDFs cultured in six-well culture plates were incubated for 24$72 \mathrm{~h}$ in $2.4 \mathrm{ml}$ of the culture medium with a mixture containing $10 \mathrm{nM}$ AHR or control siRNA and $12 \mu \mathrm{l}$ of the HiPerFect Transfection reagent (Qiagen, Hilden, Germany).

\subsection{Quantitative reverse-transcription PCR analysis}

Total RNA was extracted using the RNeasy Mini kit (Qiagen). Reverse transcription was performed using PrimeScript RT reagent kit (Takara Bio, Otsu, Japan). Quantitative reverse-transcription PCR (qRT-PCR) was conducted on a CFX Connect Real-time System (Bio-Rad, Hercules, CA) using SYBR Premix ExTaq (Takara Bio). Amplification was initiated at $95^{\circ} \mathrm{C}$ for $30 \mathrm{~s}$, followed by 40 cycles of qRT-PCR at $95^{\circ} \mathrm{C}$ for $5 \mathrm{~s}$ (denaturation) and $60^{\circ} \mathrm{C}$ for $20 \mathrm{~s}$ (annealing/extension). mRNA expression was measured in triplicate and mRNA levels normalized to ACTB levels were expressed as fold induction relative to the control group. The sequences of primers are presented in Table 1. 
Table 1

The sequences of the primers.

\begin{tabular}{|c|c|}
\hline \multirow[t]{2}{*}{$A C T B$} & sense: 5'-ATTGCCGACAGGATGCAGA-3' \\
\hline & antisense: 5'-GAGTACTTGCGCTCAGGAGGA-3' \\
\hline \multirow[t]{2}{*}{ ACTA2 } & sense: 5'-TCTGGAGATGGTGTCACCCACAAT-3' \\
\hline & antisense: 5'-AATAGCCACGCTCAGTCAGG-3' \\
\hline \multirow[t]{2}{*}{ COL1A1 } & sense: 5'-GCTTGGTCCACTTGCTTGAAGA-3' \\
\hline & antisense: 5'-GAGCATTGCCTTTGATTGCTG-3' \\
\hline \multirow[t]{2}{*}{ COL1A2 } & sense: 5'-GAGGGCAACAGCAGGTTCACTTA-3' \\
\hline & antisense: 5'-TCAGCACCACCGATGTCCAA-3' \\
\hline \multirow[t]{2}{*}{$A H R$} & sense: 5'-ATCACCTACGCCAGTCGCAAG-3' \\
\hline & antisense: 5'-AGGCTAGCCAAACGGTCCAAC-3' \\
\hline CYP1A1 & SA Biosciences ID: PPH01271E \\
\hline CYP1B1 & SA Biosciences ID: PPH00435F-200 \\
\hline
\end{tabular}

\subsection{Western blot analysis}

Total protein lysates from cells were isolated with lysis buffer (Complete Lysis-M; Roche Diagnostics, Basel, Switzerland). The protein concentrations in the lysate were measured using a BCA Protein Assay Kit (Thermo Fisher Scientific). Equal amounts of protein $(10 \mu \mathrm{g})$ were dissolved in NuPage LDS Sample Buffer (Invitrogen, Carlsbad, CA) and 10\% NuPage Sample Reducing Agent (Invitrogen). The lysates were boiled at $70^{\circ} \mathrm{C}$ for $10 \mathrm{~min}$ and loaded and run on $4 \%-12 \%$ NuPage Bis-Tris Gels (Invitrogen) at $200 \mathrm{~V}$ for $20 \mathrm{~min}$. The proteins were transferred onto polyvinylidene difluoride membranes (Invitrogen) and the membranes were blocked with Western Breeze Blocker/Diluent (Invitrogen). The membranes were probed with anti-ACTA2 antibody (1:1000 dilution), anti-collagen I antibody (1:5000 dilution), anti-AHR antibody (1:200 dilution), anti-Smad2/3 antibody (1:1000 dilution), anti-pSmad2/3 antibody (1:1000 dilution), and anti- $\beta$-actin antibody (1:1000 dilution) overnight at $4{ }^{\circ} \mathrm{C}$. HRP-conjugated antimouse or anti-rabbit IgG antibody (Cell Signaling Technology) served as a secondary antibody. The visualization of protein bands was accomplished with the SuperSignal West Pico Chemiluminescent Substrate (Thermo Fisher Scientific) using the ChemiDoc touch imaging system (Bio-Rad). Densitometric analysis of protein bands was performed using Image J software (National Institutes of Health). ImageJ is a public-domain, Java-based image processing program developed at the National Institutes of Health (Bethesda, MD).

\subsection{Cellular nuclear protein preparation for western blot analysis}

NHDFs were treated with FICZ $(100 \mathrm{nM})$ and TGF- $\beta(5 \mathrm{ng} / \mathrm{ml})$ in the absence or presence of $\mathrm{CH}-223191(10 \mu \mathrm{M})$. Cell nuclear protein was collected using an NE-PER Nuclear Cytoplasmic Extraction Reagent Kit (Thermo Fisher Scientific), in accordance with the manufacturer's instructions. The nuclear and cytoplasmic $\mathrm{pSmad} 2 / 3$ and $\mathrm{Smad} 2 / 3$ expression was analyzed by western blotting. HDAC1 and tubulin were used as internal loading controls for nuclear and cytoplasmic proteins, respectively.

\subsection{Immunofluorescence and confocal laser scanning microscopic analyses}

For the visualization of AHR, NHDFs $\left(2 \times 10^{4}\right)$ cultured on a fourwell chamber slide (Thermo Fisher Scientific) with or without FICZ were washed in phosphate-buffered saline (PBS) and fixed with acetone for $10 \mathrm{~min}$. For the detection of $\mathrm{pSmad} 2 / 3$, NHDFs cultured on slides with or without FICZ (100 nM) and TGF- $\beta(5 \mathrm{ng} / \mathrm{ml})$ were washed in PBS and then fixed. In the indicated experiments, $\mathrm{CH}$ $223191(10 \mu \mathrm{M})$ was added $1 \mathrm{~h}$ prior to the addition of other reagents. The fixed cells were then blocked with $10 \%(\mathrm{w} / \mathrm{v}) \mathrm{BSA}$ in PBS for $30 \mathrm{~min}$. The samples were incubated with an anti-AHR antibody (1:50) or anti-pSmad2/3 goat polyclonal antibody (1:50) overnight at $4^{\circ} \mathrm{C}$. The slides were washed with PBS and then incubated for $1 \mathrm{~h}$ at room temperature with a secondary antibody: a goat anti-rabbit IgG antibody conjugated with Alexa Fluor ${ }^{\circledR} 546$ (A11010; Invitrogen) or a donkey anti-goat IgG conjugated with Alexa Fluor ${ }^{\circledR} 488$ (ab150129; Abcam). After nuclear staining with 4',6-diamidino-2-phenylindole (DAPI), slides were mounted with UltraCruz $^{\mathrm{TM}}$ Mounting Medium (Santa Cruz Biotechnology). The images were acquired using a D-Eclipse confocal laser scanning microscope (Nikon, Tokyo, Japan).

\subsection{F-actin staining}

For the detection of actin polymerization, NHDFs were cultured on four-well chamber slides (Thermo Fisher Scientific) in the presence of TGF- $\beta(5 \mathrm{ng} / \mathrm{ml})$ with or without FICZ (100 nM). In the indicated experiments, $\mathrm{CH}-223191(10 \mu \mathrm{M})$ was added $1 \mathrm{~h}$ prior to the other reagents. The slides were washed with PBS, fixed with 3.7\% paraformaldehyde (Wako Pure Chemical Industries, Ltd., Osaka, Japan) for 10 min each, and blocked with $10 \%$ BSA (w/v) in PBS for 30 min. Subsequently, fluorescent Alexa Fluor ${ }^{\circledR} 488$ conjugated phalloidin (A12379; Thermo Fisher Scientific) diluted in PBS was added and then incubated for $20 \mathrm{~min}$. After nuclear staining with DAPI, slides were mounted with UltraCruz ${ }^{\mathrm{TM}}$ mounting medium (Santa Cruz Biotechnology). The images were acquired using a D-Eclipse confocal laser scanning microscope (Nikon).

\subsection{Statistical analysis}

All data are presented as mean \pm standard error (S.E.) in three independent experiments. The significance of differences between groups was assessed using Student's unpaired two-tailed $t$-test (when two groups were analyzed) or one-way analysis of variance (for three or more groups). All analyses were performed with GraphPad Prism5 software. A $P$ value of $<0.05$ was considered statistically significant.

\section{Results}

3.1. TGF- $\beta$ upregulates ACTA2 and COL1A1 expression and enhances assembly of actin stress fibers in NHDFs

First, we determined whether TGF- $\beta$ regulates the gene expression of ACTA2, COL1A1, and COL1A2 in NHDFs. NHDFs were incubated with various concentrations of TGF- $\beta(0.1,1$, and $5 \mathrm{ng} /$ $\mathrm{ml}$ ) for $24 \mathrm{~h}$. As shown in Fig. 1A, TGF- $\beta$ upregulated the expression of ACTA2 and COL1A1 in a concentration-dependent manner (see also Fig. $4 \mathrm{~B}$ for protein expression data), while TGF- $\beta$ did not change the expression of COL1A2 (Supplementary Fig. S1). In parallel with these results, immunofluorescence analyses revealed the clear assembly of actin stress fibers in NHDFs treated for $24 \mathrm{~h}$ with TGF- $\beta$ ( $5 \mathrm{ng} / \mathrm{ml})$, in contrast to the case in control NHDFs (Fig. 1B), confirming that fibroblast-to-myofibroblast transition occurred as a result of TGF- $\beta$ treatment.

\subsection{FICZ induces the upregulation of CYP1B1 expression via activation of $A H R$}

The L-tryptophan metabolite FICZ is a UV-induced photoproduct that activates cytoplasmic AHR and induces its nuclear translocation [7]. We thus evaluated the biological activity of FICZ in NHDFs by visualizing AHR. NHDFs were treated with FICZ $(100 \mathrm{nM})$ for 1 to $6 \mathrm{~h}$ and then the localization of AHR was assessed by immunofluorescence analysis. As shown in Fig. 2A, AHR was distributed mainly in the cytoplasm in control NHDFs. However, FICZ did induce the nuclear translocation of AHR $1 \mathrm{~h}$ after 
A

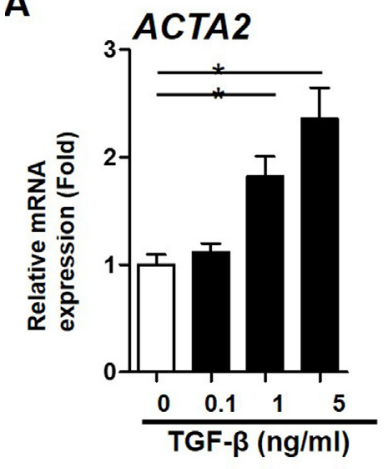

B
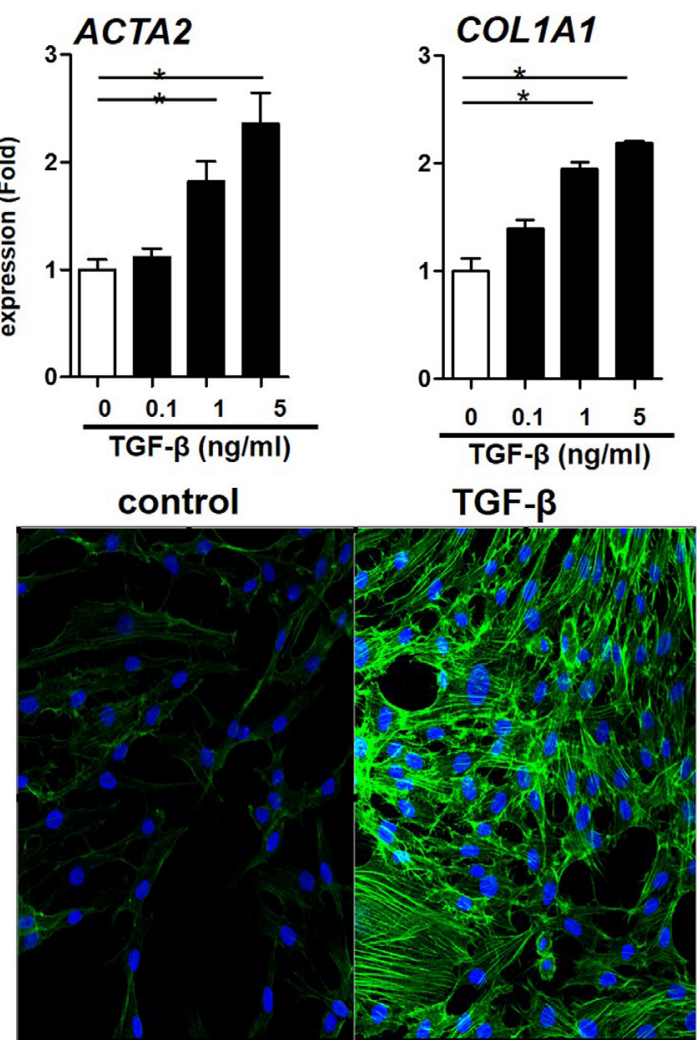

TGF- $\beta$
Fig. 1. TGF- $\beta$ upregulates the expression of ACTA2 and COL1A1 and increases the assembly of actin stress fibers in NHDFs.

(A) TGF- $\beta$ upregulated the expression of ACTA2 and COL1A1 in a dose-dependent manner in NHDFs. NHDFs were incubated in the presence of various concentrations of TGF- $\beta(0.1,1$, and $5 \mathrm{ng} / \mathrm{ml})$ for $24 \mathrm{~h}$ and then total RNA was extracted. Expression of ACTA2 and COL1A1 was measured by qRT-PCR and normalized to the expression of $A C T B$ (internal control). Data are presented as mean \pm S.E. ( $n=3$ for each group). The data are representative of experiments repeated three times with similar results. ${ }^{*} P<0.05$. (B) NHDFs treated with TGF- $\beta$ showed the clear assembly of actin stress fibers in the cytoplasm. NHDFs were treated with or without TGF- $\beta(5 \mathrm{ng} / \mathrm{ml})$ for $24 \mathrm{~h}$ and then fixed. Cells were stained with green fluorescent Alexa Fluor ${ }^{\mathbb{R}}$ 488conjugated phalloidin, subjected to nuclear staining with DAPI (blue), and visualized using a D-Eclipse confocal laser scanning microscope.

treatment (Fig. 2A). We next addressed whether FICZ activates AHR and enhances the transcription of the genes encoding the AHRspecific xenobiotic enzymes CYP1A1 and CYP1B1. NHDFs were incubated in the presence of graded concentrations of FICZ (up to $400 \mathrm{nM}$ ) for $6 \mathrm{~h}$. FICZ actually upregulated the expression of CYP1B1 in a dose-dependent manner (Fig. 2B). The biological activity of FICZ reached a plateau at $100 \mathrm{nM}$ (Fig. 2B), so we decided to use this concentration in the following experiments, unless otherwise noted. This upregulation of CYP1B1 was canceled by the presence of the selective AHR antagonist CH-223191 (Fig. 2C), which indicated that FICZ-induced CYP1B1 upregulation was dependent on AHR signaling. Interestingly, FICZ did not significantly augment the CYP1A1 expression in NHDFs (data not shown), which was in sharp contrast to the significant and preferential CYP1A1 expression in HaCaT keratinocytes (Supplementary Fig. S2).

\subsection{FICZ inhibits the TGF- $\beta$-induced upregulation of ACTA2 and COL1A1 expression}

FICZ-dependent AHR signaling regulates various biological responses other than toxicity, including immune modulation, cell differentiation, and barrier function [7,32-34]. We thus next examined whether FICZ inhibits the TGF- $\beta$-induced upregulation of ACTA2 and COL1A1 expression in NHDFs. Notably, FICZ itself

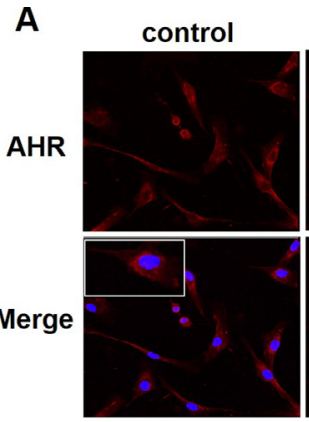

B

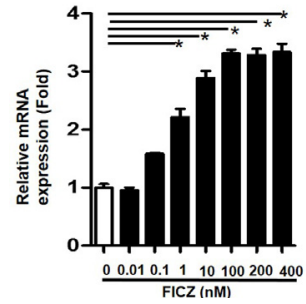

FICZ

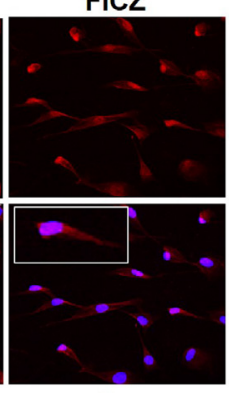

C

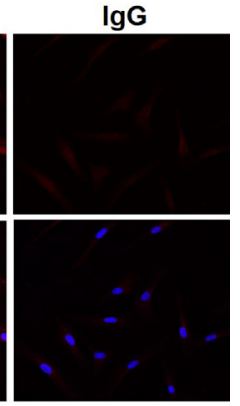

CYP1B1

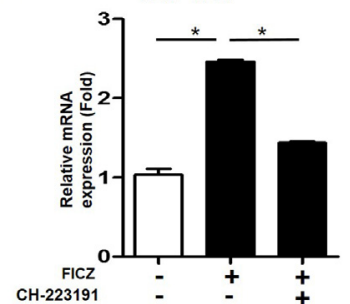

Fig. 2. FICZ induces nuclear translocation of AHR with upregulation of CYP1B1 expression.

(A) FICZ induced the translocation of AHR from the cytoplasm into the nucleus. NHDFs were treated with FICZ (100 nM) or DMSO (control) for $1 \mathrm{~h}$ and fixed. Samples were then stained with an anti-AHR antibody or isotype IgG antibody (IgG). Specific binding was detected using HRP-conjugated anti-rabbit IgG antibody followed by tyramide labeling with red fluorescent Alexa Fluor ${ }^{\mathbb{B}} 546$ for $1 \mathrm{~h}$ at room temperature. After nuclear staining with DAPI, the samples were visualized using a D-Eclipse confocal laser scanning microscope. The data are representative of experiments repeated three times with similar results. (B) FICZ upregulated the expression of CYP1B1 in a dose-dependent manner in NHDFs. NHDFs were treated with graded concentrations of FICZ $(0.01-400 \mathrm{nM})$ for $6 \mathrm{~h}$ and total RNA was extracted. CYP1B1 expression was measured by qRT-PCR and normalized to the expression of ACTB. (C) The upregulation of CYP1B1 was dependent on the AHR signal pathway. NHDFs were incubated in the presence of FICZ ( $100 \mathrm{nM})$ or DMSO (control) for $6 \mathrm{~h}$. Total RNA was extracted and CYP1B1 expression was measured by qRT-PCR. A selective AHR antagonist, $\mathrm{CH}-223191(10 \mu \mathrm{M})$, was added to the culture $1 \mathrm{~h}$ prior to the addition of FICZ. The expression levels of CYP1B1 normalized to ACTB levels are expressed as fold induction compared with the control group. (B, C) The data are representative of experiments repeated three times with similar results. ${ }^{*} P<0.05$.

decreased the basal levels of ACTA2 and COL1A1 expression in a dose-dependent manner in NHDFs (Supplementary Fig. S3). Furthermore, the TGF- $\beta$-induced upregulation of ACTA2 and COL1A1 expression was ameliorated in the presence of FICZ, which occurred in a concentration-dependent manner (Fig. 3).

\subsection{The inhibitory actions of FICZ on TGF- $\beta$-mediated ACTA2 and collagen I expression and actin filament polymerization are independent of AHR signaling}

We next investigated whether the inhibitory effects of FICZ on the TGF- $\beta$-mediated ACTA2 and collagen I expression and actin filament polymerization are AHR-dependent using selective AHR antagonists or AHR siRNA. Unexpectedly, the inhibitory action of FICZ on the TGF- $\beta$-induced upregulation of ACTA2 was not blocked by the selective AHR antagonist $\mathrm{CH}-223191$ (Fig. 4A) or GNF351 (Supplementary Fig. S4A). Western blot analysis revealed that TGF$\beta$ enhanced the protein expression of ACTA2 and collagen I, which was inhibited in the presence of FICZ (Fig. 4B). The capacity for FICZ to inhibit the TGF- $\beta$-mediated upregulation of ACTA2 and collagen I protein was not blocked by $\mathrm{CH}-223191$ (Fig. 4B) or GNF351 (Supplementary Fig. S4B). The inhibitory action of FICZ on actin filament assembly was also not canceled in the presence of $\mathrm{CH}$ 223191 (Fig. 4C-F). Additionally, the inhibitory effect of FICZ on 

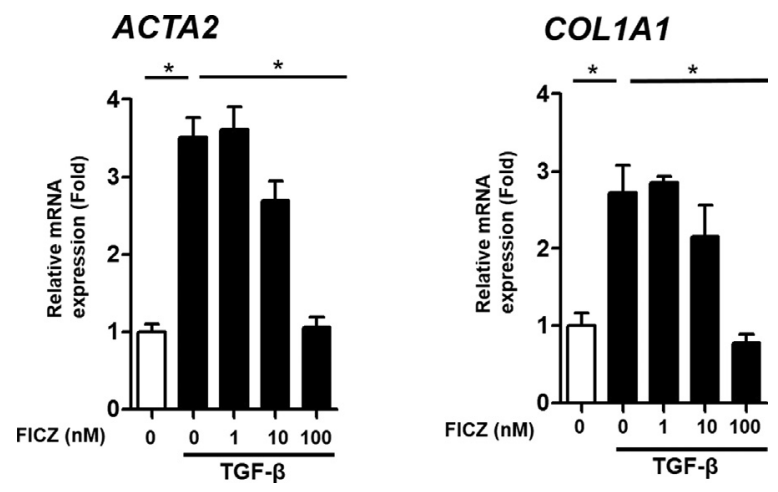

Fig. 3. FICZ inhibits TGF- $\beta$-induced upregulation of $A C T A 2$ and COL1A1 expression in NHDFs.

NHDFs were incubated with TGF- $\beta$ ( $5 \mathrm{ng} / \mathrm{ml}$ ) without or with graded concentrations of $\operatorname{FICZ}(1,10$, and $100 \mathrm{nM})$ for $24 \mathrm{~h}$ and then total RNA was extracted. Expression of ACTA2 and COL1A1 was analyzed by qRT-PCR. These expression levels were normalized to the expression of $A C T B$. The data are representative of experiments repeated three times with similar results. ${ }^{*} P<0.05$

TGF- $\beta$-regulated ACTA2 expression (Fig. 5B) and the reduced expression of ACTA2 and collagen I in the presence of FICZ (Fig. 5C, D) were not canceled in AHR siRNA-transfected NHDFs. These results indicated that FICZ inhibited the TGF- $\beta$-mediated profibrotic response or collagen maintenance in an AHR-independent fashion.
3.5. Another endogenous AHR agonist, kynurenine, also inhibits TGF$\beta$-induced ACTA2 and collagen I upregulation in NHDFs

Other endogenous AHR ligands including tryptophan metabolites, kynurenine, and ITE have been identified. Kynurenine $(50 \mu \mathrm{g} / \mathrm{ml})$ induced CYP1A1 upregulation in primary human fibroblasts isolated from foreskin samples of healthy donors via the nuclear translocation of AHR [35]. Therefore, we performed the following experiments using kynurenine $(50 \mu \mathrm{g} / \mathrm{ml})$. We investigated whether kynurenine also exerts inhibitory effects on TGF$\beta$-regulated collagen production in NHDFs. The findings showed that kynurenine inhibited TGF- $\beta$-induced ACTA2 and collagen I upregulation in an AHR-independent manner (Supplementary Fig. S5). However, its effects were very slight in comparison with those of FICZ.

\subsection{FICZ inhibits nuclear translocation of pSmad2/3}

It is well known that TGF- $\beta$ signaling is mediated by the Smad protein family. After stimulation with TGF- $\beta, \operatorname{Smad} 2 / 3$ proteins are phosphorylated and the cytoplasmic $\mathrm{pSmad} 2 / 3$ translocates into the nucleus and induces the transcription of TGF- $\beta$-responsive genes. We next investigated whether FICZ downregulates the TGF- $\beta$-induced phosphorylation of $\operatorname{Smad} 2 / 3$. As shown in Fig. 6A, TGF- $\beta$ did induce pSmad $2 / 3$ expression 5 min after TGF- $\beta$ stimulation. Notably, FICZ did not reduce the expression levels of $\mathrm{pSmad} 2 / 3$ induced by TGF- $\beta$ (Fig. 6B). In addition, the AHR antagonist $\mathrm{CH}-223191$ again did not affect the $\mathrm{pSmad} 2 / 3$ protein
A
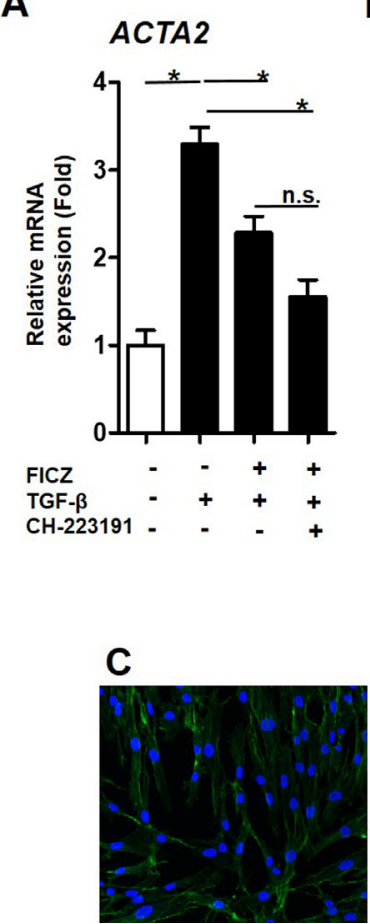

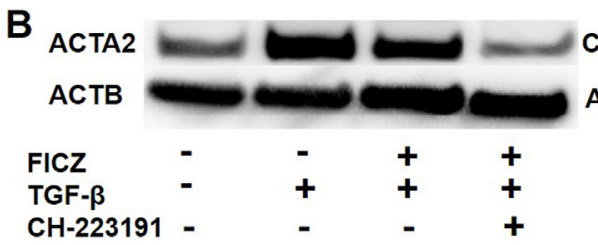

ACTA2

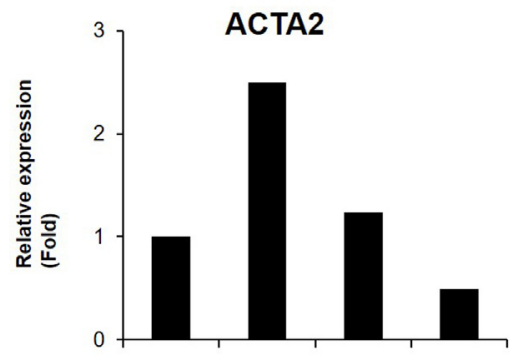

D

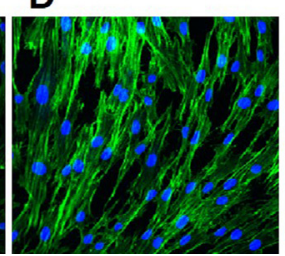

E

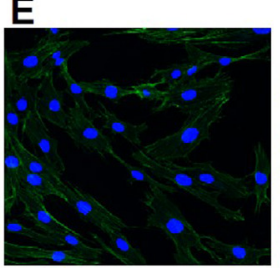

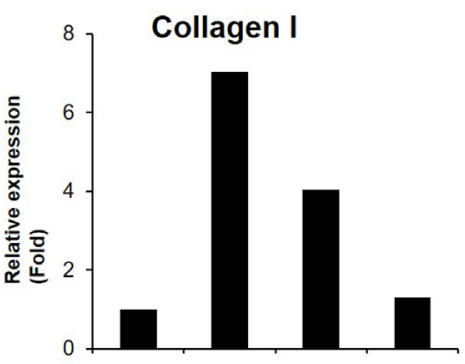
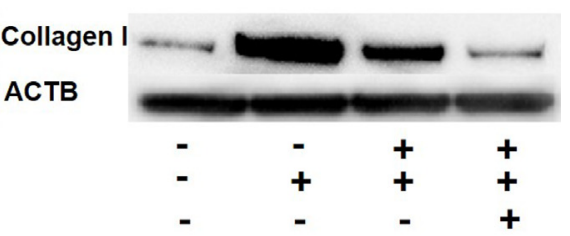

-

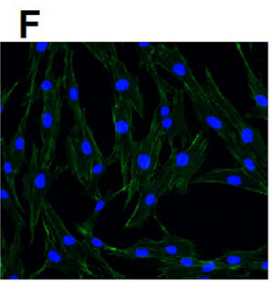

Fig. 4. FICZ inhibits TGF- $\beta$-induced upregulation of ACTA2 and collagen I expression in an AHR-independent manner.

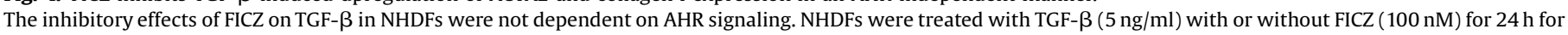

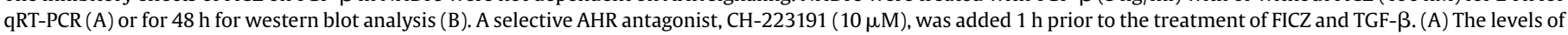

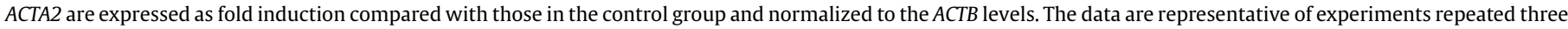

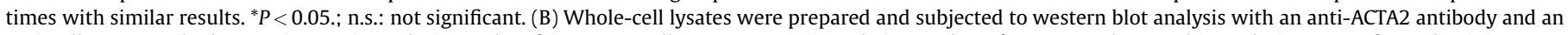

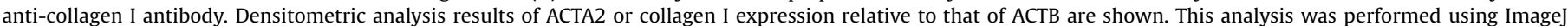

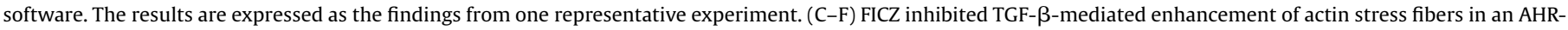

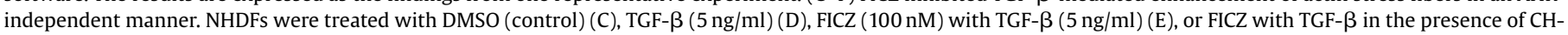

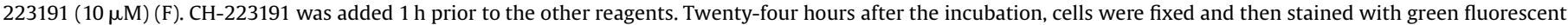

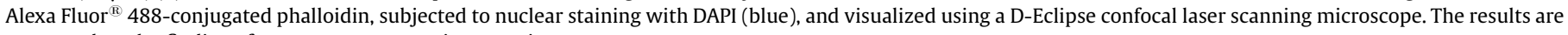
expressed as the findings from one representative experiment. 
A

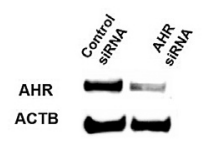

B

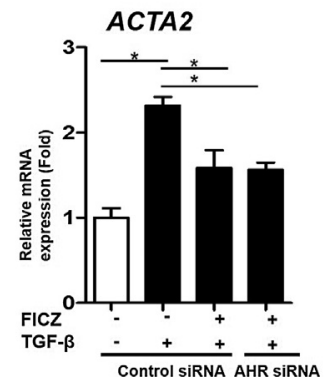

C

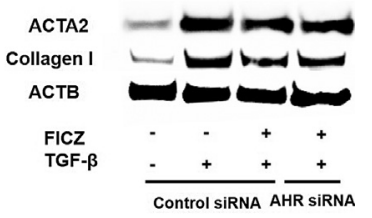

Fig. 5. The inhibitory effects of FICZ on TGF- $\beta$-induced ACTA2/collagen I downregulation are also unchanged in AHR-knockdown NHDFs.

AHR siRNA- or control siRNA-transfected NHDFs were treated with TGF- $\beta(5 \mathrm{ng} / \mathrm{ml})$ with or without FICZ $(100 \mathrm{nM})$. (A) The expression of AHR in AHR siRNA-transfected NHDFs. (B) The expression of ACTA2 in NHDFs treated with TGF- $\beta(5 \mathrm{ng} / \mathrm{ml})$ in the absence or presence of FICZ ( $100 \mathrm{nM})$ for $24 \mathrm{~h}$ was analyzed by qRT-PCR. Data are expressed as mean \pm S.E.; $\mathrm{n}=3$ for each group; ${ }^{*} P<0.05$. (C) Expression of ACTA2 and collagen I was analyzed by western blotting using an anti-ACTA2 antibody and an anti-collagen I antibody. The data are representative of experiments repeated three times with similar results.

level (Fig. 6B). We then examined whether FICZ interferes with the TGF- $\beta$-induced nuclear translocation of $\mathrm{pSmad} 2 / 3$ using western blot analysis and immunofluorescence staining. In the untreated control NHDFs, pSmad2/3 was hardly detected in both cytoplasmic and nuclear portions (Fig. 6C). After the treatment of TGF- $\beta$, $\mathrm{pSmad} 2 / 3$ was dominantly detected in the nucleus (Fig. 6C). Notably, FICZ reduced the expression of $\mathrm{pSmad} 2 / 3$ in the nucleus, while it increased that in the cytoplasm (Fig. 6C), which suggests that FICZ inhibits the nuclear translocation of $\mathrm{pSmad} 2 / 3$ induced by TGF- $\beta$. These effects were maintained irrespective of the presence or absence of $\mathrm{CH}-223191$ (Fig. 6C). Immunofluorescence analysis also showed that FICZ attenuated the staining of pSmad2/ 3 in the nucleus in the TGF- $\beta$-treated NHDFs, which was not altered in the presence of $\mathrm{CH}-223191$ (Supplementary Fig. S6). We found that FICZ exerted inhibitory effects on TGF- $\beta$-induced ACTA2 and collagen I upregulation in NHDFs via prevention of the nuclear translocation of $\mathrm{pSmad} 2 / 3$ in an AHR-independent manner.

\section{Discussion}

In photo-aged skin, major dermal alterations occur, which are characterized by disorganization and dispersion of collagen bundles [29]. UV irradiation has been shown to decrease the collagen biosynthesis by fibroblasts [30,31]. TGF- $\beta$, one of the major profibrotic cytokines, is produced in damaged skin during tissue injury and is important in wound healing [36]. It transforms fibroblasts into ACTA2-expressing myofibroblasts that produce abundant collagen I $[26,28]$. It has been demonstrated that UV irradiation impairs TGF- $\beta /$ Smad signaling, which leads to reduced collagen I production in fibroblasts [31,37].

UV irradiation induces diverse cytoplasmic and nuclear bioresponses $[1,2,38]$. Previous studies suggested that AHR signaling plays an integral part in UV-B-induced responses in human keratinocytes because UV exposure upregulates the gene expression of the AHR-specific xenobiotic-metabolizing enzymes CYP1A1 and CYP1B1 [7,19,39]. AHR is a chemical sensor that recognizes various ligands including dioxins, polyphenols, microbiota products, and numerous phytochemicals [15-17,40-42]. It has been reported that UV exposure generates diverse endogenous photosensitizers including urocanic acid, flavins, and some sterols $[5,43,44]$. FICZ is a tryptophan metabolite generated not only by UVB but also by visible light or UVA exposure $[11,19,20]$. The

A

B
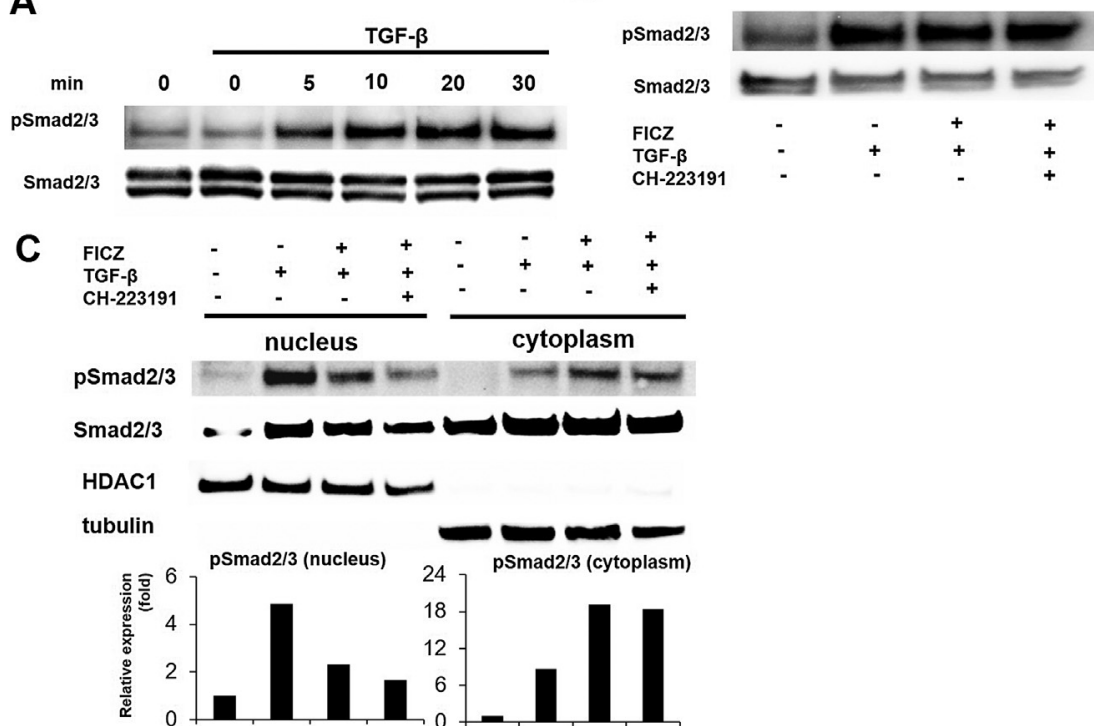

Fig. 6. FICZ prevents nuclear translocation of $\mathrm{pSmad} 2 / 3$ in the TGF- $\beta$-treated NHDFs.

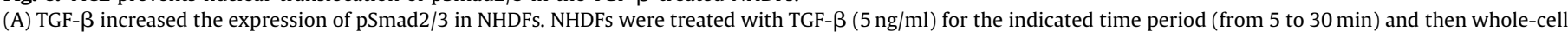

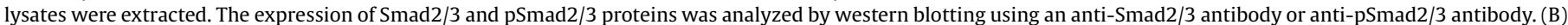

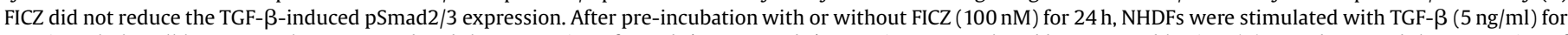

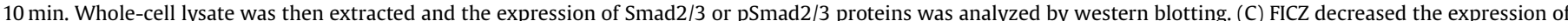

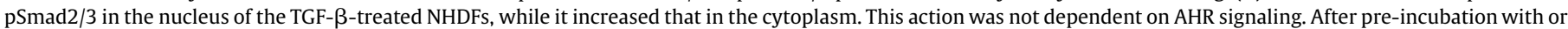

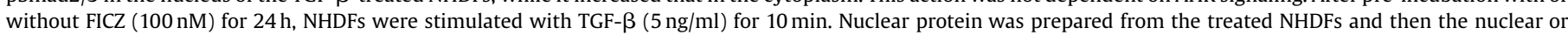

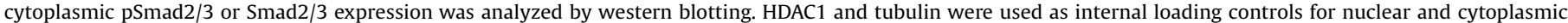

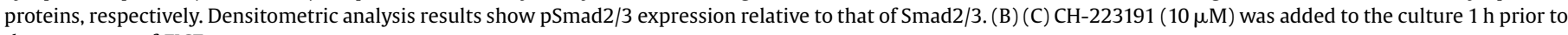
the treatment of FICZ. 
intracellular generation of FICZ has been demonstrated in UVBirradiated HaCaT keratinocytes, which was also shown to be significantly attenuated by tryptophan starvation [7]. FICZ itself is a good substrate for the metabolizing activity of CYP1A1 enzyme $[11,45]$, and the metabolites of FICZ are present in human urine [11]. Direct detection of FICZ in vivo has yet to be achieved. However, we speculate that FICZ is generated in both epidermis and dermis because the endogenous fluorescence of tryptophan presenting in the epidermis and dermis has been used for fluorescence spectroscopy to investigate human skin in vivo $[46,47]$. Notably, FICZ is a high-affinity AHR ligand and is now considered as the major photo-product to mediate upregulation of the UV-induced CYP1 family of enzymes $[7,11,19,20]$. However, the regulatory role of FICZ in TGF- $\beta$-mediated collagen production has not been fully assessed in human dermal fibroblasts.

In the present study, we confirmed that FICZ activated AHR and induced its nuclear translocation in NHDFs. Along with AHR activation, FICZ upregulated the expression of CYP1B1, but not CYP1A1, which was abrogated by the selective AHR antagonist $\mathrm{CH}-$ 223191. These results are consistent with previous studies showing that AHR signaling is cell-type-specific and that CYP1A1 is preferentially expressed in keratinocytes and hepatocytes $[20,48]$, while CYP1B1 is so in fibroblasts [48]. We also confirmed that TGF- $\beta$ significantly upregulated the transcriptional and protein levels of ACTA2 and collagen I in NHDFs with actin filament polymerization, a hallmark of fibroblast-to-myofibroblast transition [25,28]. Notably, FICZ itself decreased the basal levels of ACTA2 and COL1A1 expression in a dose-dependent manner in NHDFs. Furthermore, FICZ significantly inhibited the TGF- $\beta$-mediated upregulation of ACTA2/collagen I and the transition to myofibroblasts, but unexpectedly did so in an AHR-independent manner. TGF- $\beta$ has been reported to exert its profibrotic activity via the phosphorylation of Smad2/3 [36]. However, FICZ did not alter the pSmad $2 / 3$ levels in NHDFs induced by TGF- $\beta$. We finally revealed that FICZ inhibited the cytoplasmic-to-nuclear translocation of $\mathrm{pSmad} 2 / 3$ in NHDFs.

FICZ mediates various biological responses including immune modulation [32], circadian rhythm [49], as well as intestinal barrier function [34] and photooxidative stress [8], mostly in an AHR-dependent manner. Besides FICZ, there are tryptophan derivatives that are endogenous AHR ligands (e.g., kynurenine and ITE). Notably, these AHR ligands were also shown to modulate collagen production in fibroblasts in various organs $[13,35,50,51]$. For example, kynurenine suppressed the expression of collagen I protein in human dermal fibroblasts [35]. The present study also found that kynurenine inhibited TGF- $\beta$-induced ACTA2 and collagen I upregulation in an AHR-independent manner in NHDFs; however, its effects were insignificant. Meanwhile, ITE was shown to attenuate TGF- $\beta$-induced differentiation to myofibroblasts in human orbital, corneal, and lung fibroblasts [13]. ITE possessed similar inhibitory effects in mouse corneal and lung fibroblasts, which were independent of AHR [13]. Moreover, Woeller et al. described that both ITE and FICZ impaired TGF$\beta$-induced upregulation of ACTA2 and calponin in human orbital fibroblasts, and suppressed the production of collagen I [50]. The inhibitory effects of ITE and FICZ were also shown to be dependent on AHR because they were canceled by the transfection of AHR siRNA [50]. In intestinal fibroblasts, FICZ also inhibited the TGF- $\beta$ - or TNF- $\alpha$-induced production of collagen in an AHR-dependent manner [51]. The present study demonstrates the AHR-independent inhibitory action of FICZ on the TGF$\beta$-mediated expression of collagen I in NHDFs. These studies suggest that FICZ may inhibit TGF- $\beta$ activity in an AHR-dependent and/or AHR-independent manner with the choice between these two options depending on the fibroblast source and experimental protocol used.
Finally, we revealed that FICZ interferes with TGF- $\beta / S$ mad signaling in NHDFs. TGF- $\beta$ phosphorylates $S \operatorname{mad} 2 / 3$ and then pSmad2/3 carries the signal to the nucleus. We found that FICZ did not alter the expression of $S \operatorname{mad} 2 / 3$ or TGF- $\beta$-induced phosphorylation of $\operatorname{Smad} 2 / 3$, but did decrease pSmad2/3 levels in the nucleus, while increasing those in the cytoplasm. These results clearly showed that FICZ interferes with the TGF- $\beta$ signaling in NHDFs via prevention of the nuclear translocation of pSmad2/3. Interestingly, another tryptophan derivative, ITE, also inhibited TGF- $\beta$ signaling by preventing the nuclear translocation of Smad2/ 3 in human orbital fibroblasts [13]. Further analysis of the mechanisms involved in the inhibition of TGF- $\beta$ signaling by FICZ is warranted.

In conclusion, we here demonstrate that the tryptophan photoproduct FICZ inhibits TGF- $\beta$-induced collagen production in human dermal fibroblasts in an AHR-independent manner. The FICZ-mediated inhibition of collagen synthesis may be involved in UV-induced photo-aging. The targeting of FICZ signaling may thus open up a new therapeutic avenue to protect against photo-aging.

\section{Funding source}

This work was partly supported by a grant from the Japanese Ministry of Health, Labour, and Welfare (Research on Food Safety; H27-Designated Research-017).

\section{Conflict of interest}

The authors have no conflicts of interest to declare.

\section{References}

[1] Y. Devary, C. Rosette, J.A. DiDonato, M. Karin, NF-кB activation by ultraviolet light not dependent on a nuclear signal, Science 261 (1993) 1442-1445.

[2] K. Bender, C. Blattner, A. Knebel, M. Iordanov, P. Herrlich, H.J. Rahmsdorf, UVinduced signal transduction, J. Photochem. Photobiol. B 37 (1997) 1-17.

[3] G.T. Wondrak, M.K. Jacobson, E.L. Jacobson, Endogenous UVAphotosensitizers: mediators of skin photodamage and novel targets for skin photoprotection, Photochem. Photobiol. Sci. 5 (2006) 215-237.

[4] K. Sharffetter-Kochanek, M. Wlaschek, P. Brenneisen, M. Schauen, R. Blaudschun, J. Wenk, UV-induced reactive oxygen species in photocarcinogenesis and photoaging, Biol. Chem. 378 (1997) 1247-1257.

[5] K.M. Hanson, J.D. Simon, Epidermal trans-urocanic acid and the UV-A-induced photoaging of the skin, Proc. Natl. Acad. Sci. U. S. A. 95 (1998) 10576-10578.

[6] J. Baier, T. Maisch, M. Maier, M. Landthaler, W. Bäumier, Direct detection of singlet oxygen generated by UVA irradiation in human cells and skin, J. Invest. Dermatol. 127 (2007) 1498-1506.

[7] E. Fritsche, C. Schäfer, C. Calles, T. Bernsmann, T. Bernshausen, M. Wurm, et al., Lightening up the UV response by identification of the arylhydrocarbon receptor as a cytoplasmic target for ultraviolet B radiation, Proc. Natl. Acad. Sci. U. S. A. 104 (2007) 8851-8856.

[8] S.L. Park, R. Justiniano, J.D. Williams, C.M. Cabello, S. Qiao, G.T. Wondrak, The tryptophan-derived endogenous aryl hydrocarbon receptor ligand 6formylindolo[3,2-b]carbazole (FICZ) is a nanomolar UVA-photosensitizer in epidermal keratinocytes, J. Invest. Dermatol. 135 (2015) 1649-1658.

[9] L.P. Nguyen, C.A. Bradfield, The search for endogenous activators of the aryl hydrocarbon receptor, Chem. Res. Toxicol. 21 (2008) 102-116.

[10] J. Mimura, Y. Fujii-Kuriyama, Functional role of AhR in the expression of toxic effects by TCDD, Biochim. Biophys. Acta 1619 (2003) 263-268.

[11] E. Wincent, N. Amini, S. Luecke, H. Glatt, J. Bergman, C. Crescenzi, et al., The suggested physiologic aryl hydrocarbon receptor activator and cytochrome P4501 substrate 6-formylindolo[3,2-b]carbazole is present in humans, J. Biol. Chem. 284 (2009) 2690-2696.

[12] C.A. Opitz, U.M. Litzenburger, F. Sahm, M. Ott, I. Tritschler, S. Trump, et al., An endogenous tumor-promoting ligand of the human aryl hydrocarbon receptor, Nature 478 (2011) 197-203.

[13] G.M. Lehmann, X. Xi, A.A. Kulkarni, K.C. Olsen, S.J. Pollock, C.J. Baglole, et al., The aryl hydrocarbon receptor ligand ITE inhibits TGFß1-induced human myofibroblast differentiation, Am. J. Pathol. 178 (2011) 1556-1567.

[14] J. Krutmann, A. Morita, J.H. Chung, Sun exposure: what molecular photodermatology tells us about its good and bad sides, J. Invest. Dermatol. 132 (2012) 976-984

[15] G. Tsuji, M. Takahara, H. Uchi, S. Takeuchi, C. Mitoma, Y. Moroi, et al., An environmental contaminant, benzo(a)pyrene, induces oxidative stressmediated interleukin- 8 production in human keratinocytes via the aryl hydrocarbon receptor signaling pathway, J. Dermatol. Sci. 62 (2011) 42-49. 
[16] G. Tsuji, M. Takahara, H. Uchi, T. Matsuda, T. Chiba, S. Takeuchi, et al., Identification of ketoconazole as an AhR-Nrf2 activator in cultured human keratinocytes: the basis of its anti-inflammatory effect, J. Invest. Dermatol. 132 (2012) 59-68.

[17] M. Furue, M. Takahara, T. Nakahara, H. Uchi, Role of AhR/ARNT system in skin homeostasis, Arch. Dermatol. Res. 306 (2014) 769-779.

[18] M. Furue, G. Tsuji, C. Mitoma, T. Nakahara, T. Chiba, S. Morino-Koga, et al., Gene regulation of filaggrin and other skin barrier proteins via aryl hydrocarbon receptor, J. Dermatol. Sci. 80 (2015) 83-88.

[19] Y.D. Wei, U. Rannug, A. Rannug, UV-induced CYP1A1 gene expression in human cells is mediated by tryptophan, Chem. Biol. Interact. 118 (1999) 127 140.

[20] S. Nair, V.D. Kekatpure, B.L. Judson, A.B. Rifkind, R.D. Granstein, J.O. Boyle, et al., UVR exposure sensitizes keratinocytes to DNA adduct formation, Cancer Prev. Res. (Philadelphia) 2 (2009) 895-902.

[21] J. Tigges, T. Haarmann-Stemmann, C. Vogel, A. Grindel, U. Hübenthal, H. Brenden, et al., The new aryl hydrocarbon receptor antagonist E/Z-2benzylindene-5,6-dimethoxy-3,3-dimethylindan-1-one protects against UVBinduced signal transduction, J. Invest. Dermatol. 134 (2014) 556-559.

[22] K. Takei, A. Hashimoto-Hachiya, M. Takahara, G. Tsuji, T. Nakahara, M. Furue, Cynaropicrin attenuates UVB-induced oxidative stress via the AhR-Nrf2-Nqo1 pathway, Toxicol. Lett. 234 (2015) 74-80.

[23] L.T. Smith, K.A. Holbrook, J.A. Madri, Collagen types I II, and V in human embryonic and fetal skin, Am. J. Anat. 175 (1986) 507-521.

[24] J. Uitto, Biology of dermal cells and extracellular matrix, in: T. Fitzpatrick, A. Eisen, K. Wolff, I. Freedberg, K. Austin (Eds.), Dermatology in General Medicine, McGraw-Hill, New York, 1993, pp. 299-314.

[25] A. Desmoulière, C. Chaponnier, G. Gabbiani, Tissue repair, contraction, and the myofibroblast, Wound Repair Regen. 13 (2005) 7-12.

[26] B. Hinz, S.H. Phan, V.J. Thannickal, A. Galli, M.L. Bochaton-Piallat, G. Gabbiani, The myofibroblast: one function multiple origins, Am. J. Pathol. 170 (2007) 1807-1816.

[27] A. Leask, Getting out of a sticky situation: targeting the myofibroblast in scleroderma, Open Rheumatol. J. 6 (2012) 163-169.

[28] A. Desmoulière, A. Geinoz, F. Gabbiani, G. Gabbiani, Transforming growth factor-beta 1 induces alpha-smooth muscle actin expression in granulation tissue myofibroblasts and in quiescent and growing cultured fibroblasts, J. Cell Biol. 122 (1993) 103-111.

[29] R. Warren, V. Gartstein, A.M. Kligman, W. Montagna, R.A. Allendorf, G.M. Ridder, Age, sunlight, and facial skin: a histologic and quantitative study, J. Am. Acad. Dermatol. 25 (1991) 751-760.

[30] C.P. Choi, Y.I. Kim, J.W. Lee, M.H. Lee, The effect of narrowband ultraviolet B on the expression of matrix metalloproteinase-1, transforming growth factor- $\beta 1$ and type I collagen in human skin fibroblasts, Clin. Exp. Dermatol. 32 (2007) 180-185.

[31] T. Quan, T. He, S. Kang, J.J. Voorhees, G.J. Fisher, Solar ultraviolet irradiation reduces collagen in photoaged human blocking transforming growth factorbeta type II receptor/Smad signaling, Am. J. Pathol. 165 (2004) 741-751.

[32] M.D. Hayes, V. Ovcinnikovs, A.G. Smith, I. Kimber, R.J. Dearman, The aryl hydrocarbon receptor: differential contribution to T helper 17 and T cytotoxic 17 cell development, PLoS One 9 (2014) e106955, doi:http://dx.doi.org/ 10.1371 /journal.pone.0106955.

[33] P. Di Meglio, J.H. Duarte, H. Ahlfors, N.D. Owens, Y. Li, F. Villanova, et al. Activation of the aryl hydrocarbon receptor dampens the severity of inflammatory skin conditions, Immunity 40 (2014) 989-1001.

[34] B. Han, B. Sheng, Z. Zhang, A. Pu, J. Yin, Q. Wang, et al., Aryl hydrocarbon receptor activation in intestinal obstruction ameliorates intestinal barrier dysfunction via suppression of MLCK-MLC phosphorylation pathway, Shock 46 (2016) 319-328.
[35] M.S. Poormasjedi-Meibod, S. Salimi Elizei, V. Leung, R. Baradar Jalili, F. Ko, A Ghahary, Kynurenine modulates MMP-1 and type-I collagen expression via aryl hydrocarbon receptor activation in dermal fibroblasts, J. Cell Physiol. 231 (2016) 2749-2760.

[36] M. Morikawa, R. Derynck, K. Miyazono, TGF- $\beta$ and the TGF- $\beta$ family: contextdependent roles in cell and tissue physiology, Cold Spring Harb. Perspect. Biol. 8 (2016) a021873, doi:http://dx.doi.org/10.1101/cshperspect.a021873.

[37] T. Quan, T. He, J.J. Voorhees, G.J. Fisher, Ultraviolet irradiation induces Smad7 via induction of transcription factor AP-1 in human skin fibroblasts, J. Biol. Chem. 280 (2005) 8079-8085.

[38] D. Kulms, B. Pöppelmann, D. Yarosh, T.A. Luger, J. Krutmann, T. Schwarz, Nuclear and cell membrane effects contribute independently to the induction of apoptosis in human cells exposed to UVB radiation, Proc. Natl. Acad. Sci. U. S. A. 96 (1999) 7974-7979.

[39] S.K. Katiyar, M.S. Matsui, H. Mukhtar, Ultraviolet-B exposure of human skin induces cytochromes P450 1A1 and 1B1, J. Invest. Dermatol. 114 (2000) 328333.

[40] P. Magiatis, P. Pappas, G. Gaitanis, N. Mexia, E. Melliou, M. Galanou, et al. Malassezia yeasts produce a collection of exceptionally potent activators of the $\mathrm{Ah}$ (dioxin) receptor detected in diseased human skin, J. Invest. Dermatol. 133 (2013) 2023-2030.

[41] K. Takei, C. Mitoma, A. Hashimoto-Hachiya, H. Uchi, M. Takahara, G. Tsuji, et al., Antioxidant soybean tar glyteer rescues T-helper-mediated downregulation of filaggrin expression via aryl hydrocarbon receptor, J. Dermatol. 42 (2015) 171 180.

[42] K. Takei, C. Mitoma, A. Hashimoto-Hachiya, M. Takahara, M. Takahara, G. Tsuji, et al., Galactomyces fermentation filtrate prevents T helper 2-mediated reduction of filaggrin in an aryl hydrocarbon receptor-dependent manner, Clin. Exp. Dermatol. 40 (2015) 786-793.

[43] G. Viteri, A.M. Edwards, J. De la Fuente, E. Silva, Study of the interaction between triplet riboflavin and the alpha-, betaH- and betaL-crystallins of the eye lens, Photochem. Photobiol. 77 (2003) 535-540.

[44] P.W. Albro, P. Bilski, J.T. Corbett, J.L. Schroeder, C.F. Chignell, Photochemical reactions and phototoxicity of sterols: novel self-perpetuating mechanisms for lipid photooxidation, Photochem. Photobiol. 66 (1997) 316-325.

[45] L. Bergander, E. Wincent, A. Rannug, M. Foroozesh, W. Alworth, U. Rannug, Metabolic fate of the Ah receptor ligand 6-formylindolo[3,2-b]carbazole, Chem. Biol. Interact. 149 (2004) 151-164.

[46] P.R. Bargo, S.T. Walston, M. Chu, I. Seo, N. Kollias, Non-invasive assessment of tryptophan fluorescence and confocal microscopy provide information on skin barrier repair dynamics beyond TEWL, Exp. Dermatol. 22 (2013) 18-23.

[47] R. Gillies, G. Zonios, R.R. Anderson, N. Kollias, Fluorescence excitation spectroscopy provides information about human skin in vivo, J. Invest. Dermatol. 115 (2000) 704-707.

[48] S.R. Beedanagari, R.T. Taylor, O. Hankinson, Differential regulation of the dioxin-induced Cyp1a1 and Cyp1b1 genes in mouse hepatoma and fibroblast cell lines, Toxicol. Lett. 194 (2010) 26-33.

[49] M. Mukai, S.A. Tischkau, Effects of tryptophan photoproducts in the circadian timing system: searching for a physiological role for aryl hydrocarbon receptor, Toxicol. Sci. 95 (2007) 172-181.

[50] C.F. Woeller, E. Roztocil, C.L. Hammond, S.E. Feldon, R.P. Phipps, The aryl hydrocarbon receptor and its ligands inhibit myofibroblast formation and activation: implications for thyroid eye disease, Am. J. Pathol. 186 (2016) 31893202.

[51] I. Monteleone, F. Zorzi, I. Marafini, D. Di Fusco, V. Dinallo, R. Caruso, et al., Aryl hydrocarbon receptor-driven signals inhibit collagen synthesis in the gut, Eur. J. Immunol. 46 (2016) 1047-1057. 
Supplementary Fig. S1

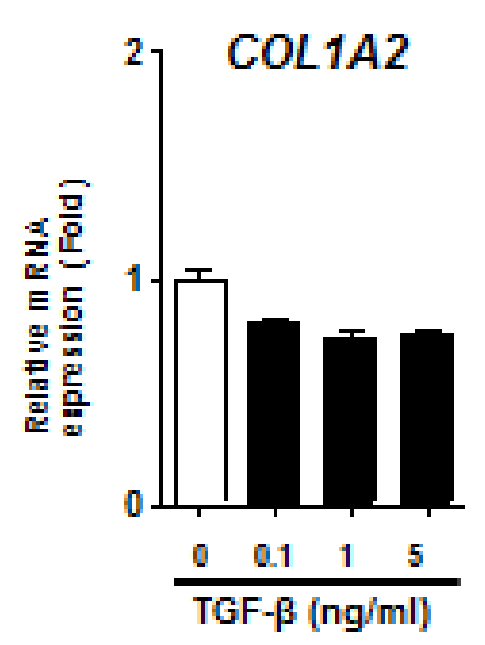

Fig. Sl. TGF- $\beta$ does not change the expression of COL1A2 in NHDFs NHDFs were incubated in the presence of various concentrations of TGF- $\beta(0.1,1$, and 5 $\mathrm{ng} / \mathrm{ml}$ ) for $24 \mathrm{~h}$ and then total RNA was extracted. Expression of $C O L 1 A 2$ was analyzed by $q R T-P C R$ and normalized to the expression of $A C T B$. Data are presented as mean \pm S.E. ( $\mathrm{n}=3$ for each group). The data are representative of experiments repeated three times with similar results. ${ }^{*} P<0.05$. 
Supplementary Fig. S2

CYP1A1

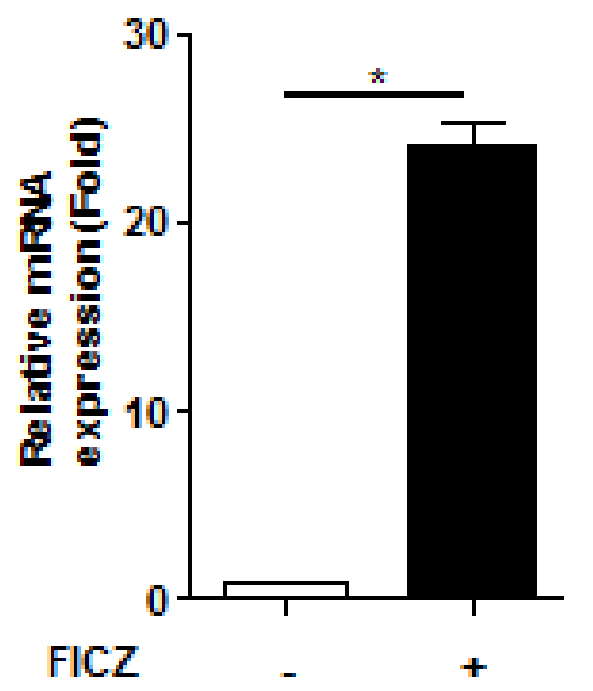

CYP1B1

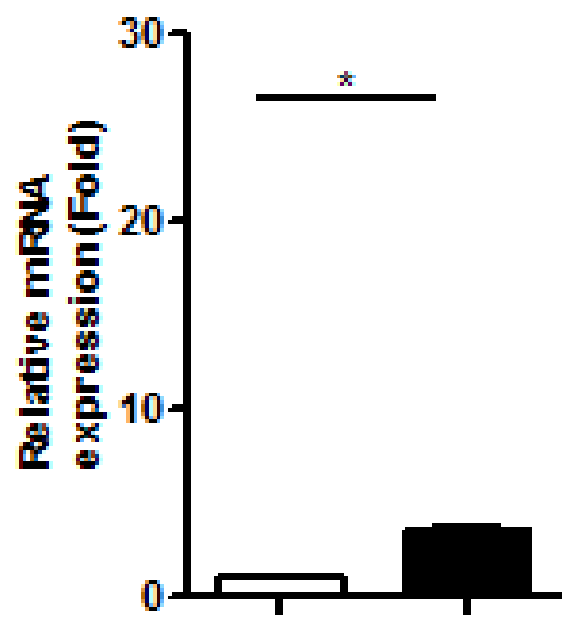

FICZ

Fig. S2. FICZ increases the expression of CYP1A1, but does not change the CYP1B1 levels in human keratinocyte $\mathrm{HaCaT}$ cells

The human keratinocyte cells $\mathrm{HaCaT}$ were treated with FICZ (100 nM) for $6 \mathrm{~h}$ and then total RNA was extracted. The expression of CYP $I A 1$ and $C Y P 1 B 1$ was measured by $q R T-P C R$ and normalized to the expression of $A C T B$. The data are representative of experiments repeated three times with similar results. ${ }^{*} P<0.05$. 
Supplementary Fig. S3
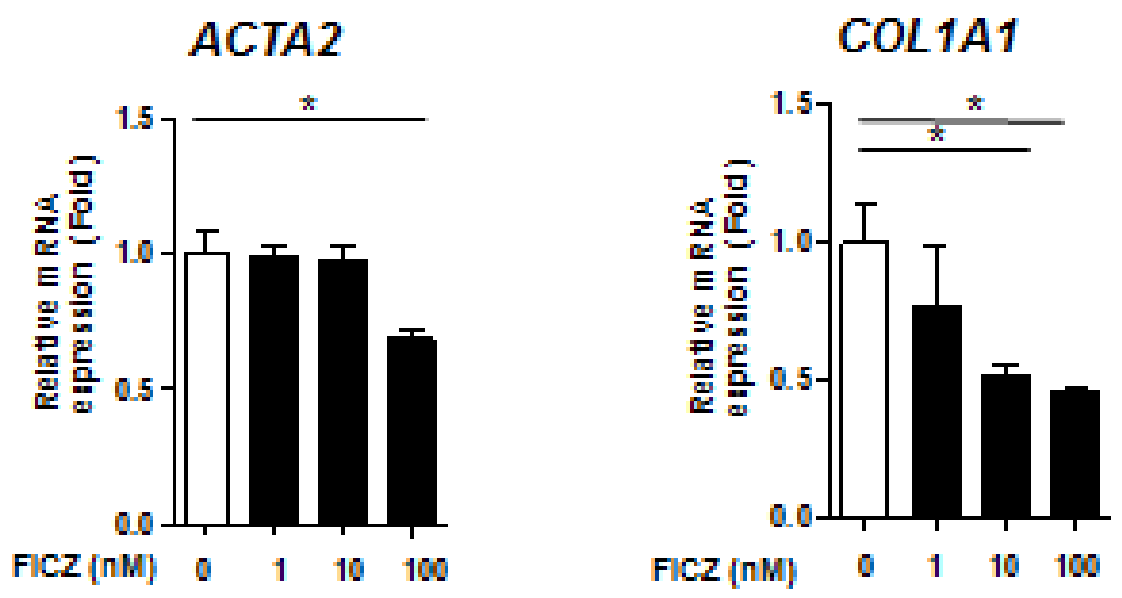

Fig. S3. FICZ itself reduces the basal expression of $A C T A 2$ and COL1A1 in a dose-dependent manner

NHDFs were incubated in the presence of various concentrations of FICZ $(1-100 \mathrm{nM})$ for $24 \mathrm{~h}$ and then total RNA was extracted. The expression of $A C T A 2$ and $C O L 1 A 1$ was analyzed by $q$ RT-PCR and normalized to the expression of $A C T B$. The data are representative of experiments repeated three times with similar results. ${ }^{*} P<0.05$. 
B

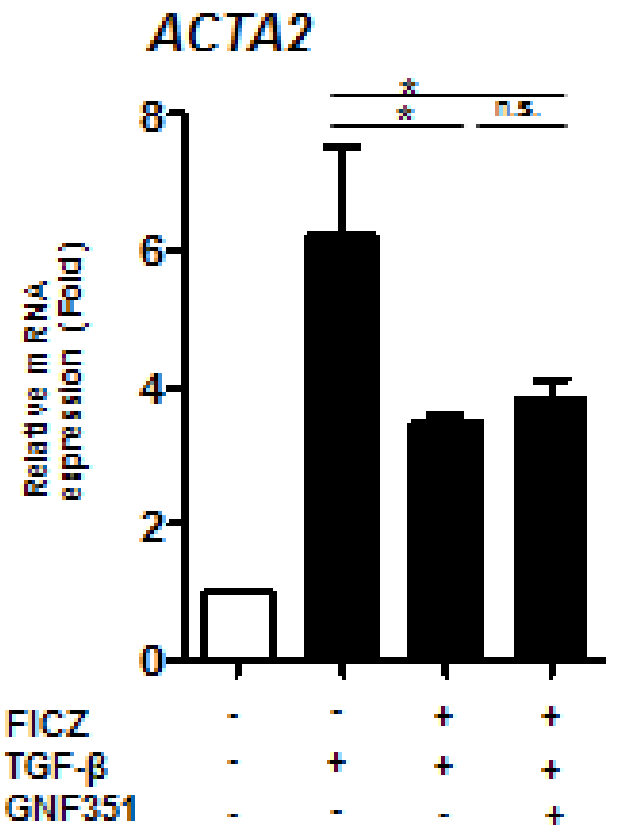

Supplementary Fig. S4

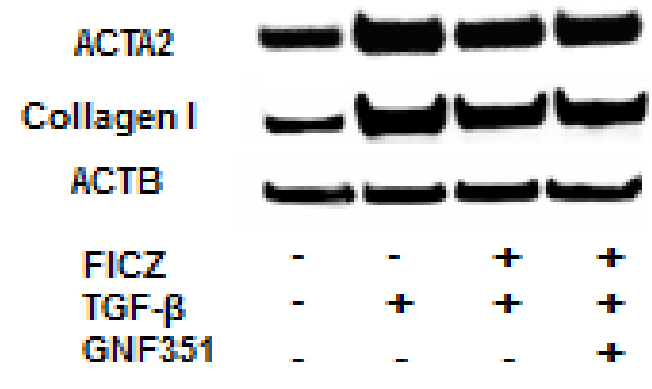

Fig. S4. The inhibitory effects of FICZ on TGF- $\beta$ are not changed in the presence of another AHR antagonist, GNF351

NHDFs were treated with TGF- $\beta(5 \mathrm{ng} / \mathrm{ml})$ with or without FICZ (100 $\mathrm{nM}$ ) for $24 \mathrm{~h}$ for $\mathrm{qRT}-\mathrm{PCR}$ analysis (A) or for $48 \mathrm{~h}$ for western blot analysis (B). A selective AHR antagonist, GNF351 (500 nM), was added $1 \mathrm{~h}$ prior to the treatment of $F I C Z$ and TGF- $\beta$. (A) The expression of $A C T A 2$ was analyzed by $q R T-P C R$ and normalized to the expression of $A C T B$. (B) Whole-cell lysates were prepared and subjected to western blot analysis with anti-ACTA2 antibody and anti-collagen I antibody. The data are representative of experiments repeated three times with similar results. ${ }^{*} P<0.05$; n.s.: not significant. 
Supplementary Fig. S5

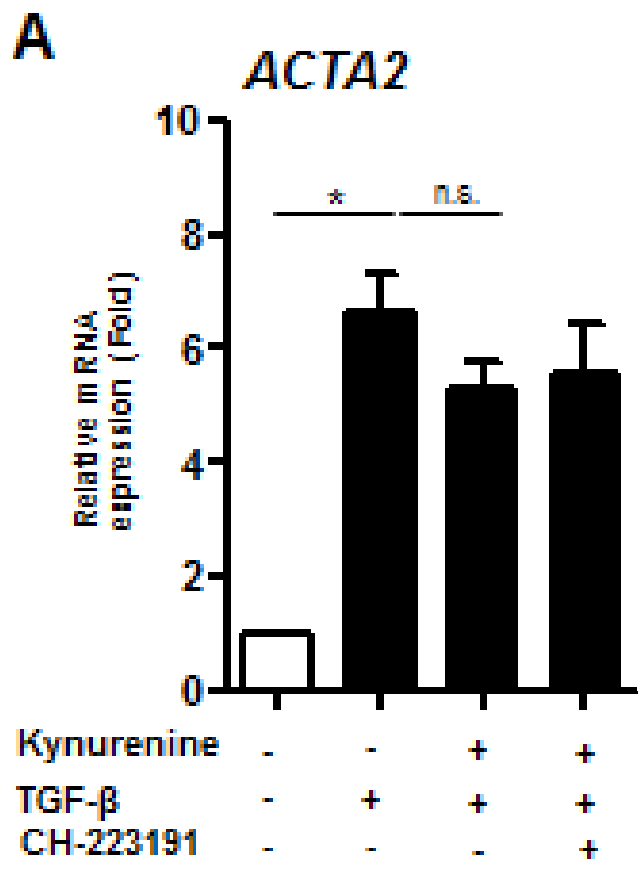

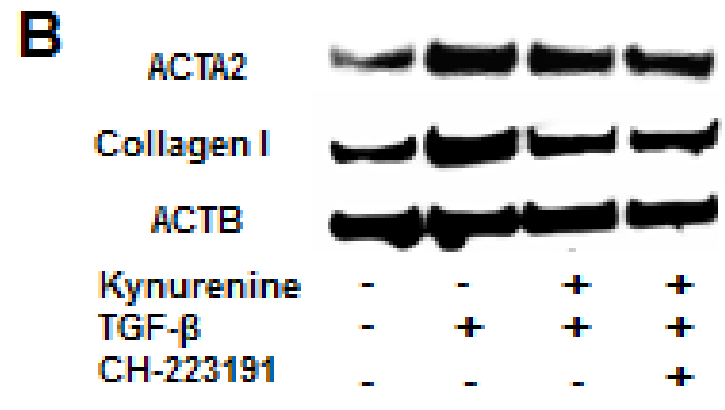

Fig. S5. An endogenous AHR agonist, kynurenine, slightly inhibits TGF- $\beta$-induced upregulation of ACTA2 and collagen I expression in an AHRindependent manner

NHDFs were treated with TGF $-\beta(5 \mathrm{ng} / \mathrm{ml})$ with or without kynurenine $(50 \mu \mathrm{g} / \mathrm{ml})$ for $24 \mathrm{~h}$ for $q R T-P C R$ analyses (A) or for $48 \mathrm{~h}$ for western blot analyses (B). $\mathrm{CH}-223191(10 \mu \mathrm{M})$ was added $1 \mathrm{~h}$ prior to the treatment of kynurenine and TGF- $\beta$. (A) The expression of ACTA2 were measured by $q R T-P C R$ and normalized to the expression of $A C T B$. (B) Whole-cell lysates were prepared and subjected to western blot analyses with an anti-ACTA2 antibody and anti-collagen I antibody. The data are representative of experiments repeated three times with similar results. ${ }^{*} P<0.05$; n.s.: not significant 
Supplementary Fig. S6

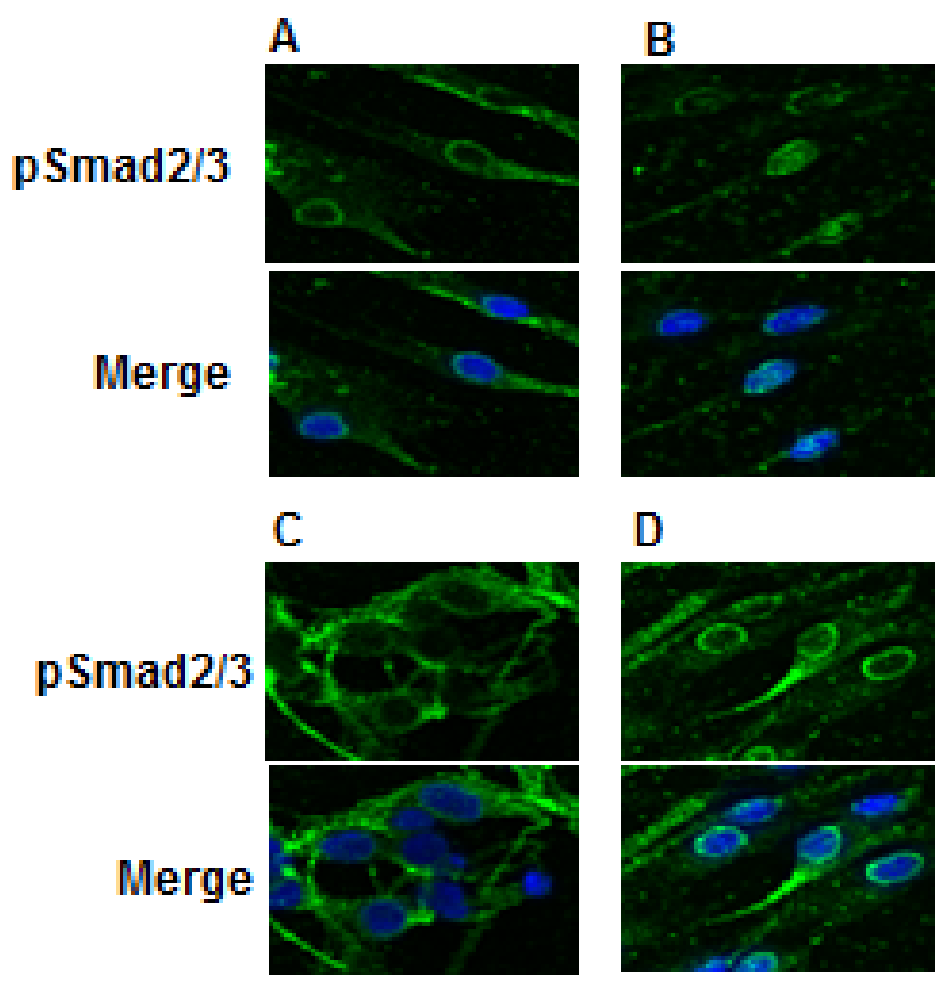

Fig. S6. FICZ prevents the nuclear translocation of $\mathrm{pSmad} 2 / 3$ induced by TGF- $\beta$

After pre-incubation with $\mathrm{FICZ}$ (100 $\mathrm{nM}$ ) for $24 \mathrm{~h}, \mathrm{NHDF}$ s were stimulated with TGF- $\beta(5 \mathrm{ng} / \mathrm{ml})$ for $1 \mathrm{~h}$. NHDFs treated with DMSO (control) (A), TGF$\beta(5 \mathrm{ng} / \mathrm{ml})$ (B), FICZ (100 nM) with TGF- $\beta$ (5 $\mathrm{ng} / \mathrm{ml})(\mathrm{C})$, or FICZ with TGF- $\beta$ in the presence of CH-223191 $(10 \mu \mathrm{M})(\mathrm{D})$. $\mathrm{CH}-223191$ was added to the culture $1 \mathrm{~h}$ prior to the treatment of FICZ. Samples were then stained with an anti-pSmad $2 / 3$ antibody (primary antibody). Specific binding was detected using HRP-conjugated antigoat $\mathrm{IgG}$ antibody followed by tyramide labeling with green fluorescent Alexa Fluor ${ }^{2} 488$ for $1 \mathrm{~h}$ at room temperature. After nuclear staining with DAPI, the samples were visualized using a $\mathrm{D}$-Eclipse confocal laser scanning microscope. 